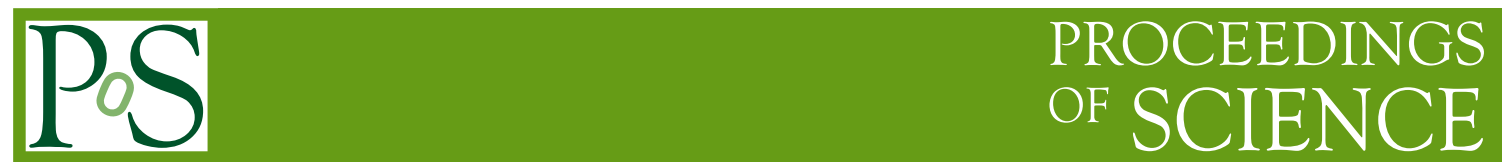

\title{
Non-forward BFKL at NLO
}

\author{
Victor Fadin*i \\ Budker INP, Novosibirsk, Russia \\ E-mail: fadin@inp.nsk.su
}

Structure of the kernel of the BFKL equation in the next-to-leading order is discussed. The twogluon contribution to the kernel for non-zero momentum transfer is considered. Different forms of this contribution are suggested. Their properties are analyzed. The quark contribution to the kernel is transformed from momentum representation to coordinate one.

Diffraction 06, International Workshop on Diffraction in High-Energy Physics September 5-10, 2006

Adamantas, Milos island, Greece

\footnotetext{
* Speaker.

${ }^{\dagger}$ Work supported in part by Russian Fund of Fundamental Researches.
} 


\section{Introduction}

The BFKL approach [1] gives a common basis for theoretical description of high energy processes with fixed (not growing with energy $\sqrt{s}$ ) momentum transfers. Talking about BFKL one usually has in mind the case of forward scattering, i.e. $t=0$ and vacuum quantum numbers in the $t$-channel. However, the BFKL approach is not limited to this particular case and, what is more, from the beginning it was developed for arbitrary $t$ and for all possible $t$-channel colour states.

Now the BFKL approach is developed in the next-to-leading approximation (NLA). In particular, the kernel of the BFKL equation for the forward scattering was found in the next-to-leading order (NLO) a long time ago [2]. But the forward kernel can carry only restrictive information about the BFKL dynamics. Moreover, the non-forward case has an advantage of smaller sensitivity to large-distance contributions, since the diffusion in the infrared region is limited by $\sqrt{|t|}$. However, calculation of the non-forward kernel at NLO was completed only recently [3]. The reason was the complexity of the two-gluon contribution.

\section{Structure of the BFKL kernel}

For any colour group representation $\mathscr{R}$ in the $t$-channel the kernel is given by the sum of "virtual" and "real" parts [4]

$$
\hat{\mathscr{K}}=\hat{\omega}_{1}+\hat{\omega}_{2}+\hat{\mathscr{K}}_{r} .
$$

The "virtual" part is universal (i.e. it does not depend on $\mathscr{R}$ ) and is expressed through the NLO gluon Regge trajectory $\omega(t)$ [5]. The "real" part is related to particle production in ReggeonReggeon collisions and consists of one-gluon, two-gluon and quark-antiquark contributions:

$$
\hat{\mathscr{K}}_{r}=\hat{\mathscr{K}}_{G}+\hat{\mathscr{K}}_{Q \bar{Q}}+\hat{\mathscr{K}}_{G G}
$$

The first part is also universal, apart from a colour coefficient, and is also known in the NLO since long ago [6].

The new contributions which appear in the NLO are $\hat{\mathscr{K}}_{Q \bar{Q}}$ and $\hat{\mathscr{K}}_{G G}$. Each of them is written as a sum of two terms with coefficients depending on a colour representation $\mathscr{R}$ in the $t$-channel. For the $Q \bar{Q}$ case both these terms are known long ago as well [7]. Instead, till recently only the piece related to the gluon channel was known for the $G G$ case [6].

Thus, the two-gluon contribution for a long time was the only missing piece in the non-forward BFKL kernel.

\section{The two-gluon contribution}

\subsection{Decomposition of the kernel}

The "non-subtracted" contribution to the kernel $\mathscr{K}_{G G}$ is written as

$$
\sum_{G_{1} G_{2}} \int \gamma^{G_{1} G_{2}}\left(\gamma^{\prime G_{1} G_{2}}\right)^{*} d \phi_{G_{1} G_{2}}
$$


where $\gamma^{G_{1} G_{2}}$ and $\gamma^{\prime G_{1} G_{2}}$ are effective vertices for two-gluon production in collision of Reggeized gluons with momenta $q_{1},-q_{2}$ and $q_{1}^{\prime},-q_{2}^{\prime}$ respectively;

$$
q_{1}-q_{1}^{\prime}=q_{2}-q_{2}^{\prime}=q, \quad q_{1}-q_{2}=q_{1}^{\prime}-q_{2}^{\prime}=k_{1}+k_{2},
$$

$q$ is the total momentum transfer, $k_{i}$ are momenta of produced gluons, $d \phi_{G_{1} G_{2}}$ is their phase space element; the sum goes over polarizations and colours of produced gluons.

For two-gluon states the integral over their invariant mass $k^{2}$ is logarithmically divergent at large $k^{2}$, that requires subtraction of the region of large invariant mass. This region is taken into account in the leading terms.

The two-gluon vertex [8] contains two colour structures:

$$
\gamma^{G_{1} G_{2}}=T^{G_{1}} T^{G_{2}} \gamma_{12}+T^{G_{2}} T^{G_{1}} \gamma_{21}
$$

Accordingly, for any representation $\mathscr{R}$ of the colour group the two-gluon contribution $\mathscr{K}_{G G}$ contains two terms: "direct", corresponding to the colour factor $T^{G_{1}} T^{G_{2}} T^{G_{2}} T^{G_{1}}$, and "interference", corresponding to the colour factor $T^{G_{1}} T^{G_{2}} T^{G_{1}} T^{G_{2}}$, with different colour coefficients $a_{R}$ and $b_{R}$ and the functions $F_{a}$ and $F_{b}$,

$$
F_{a} \propto \gamma_{1} \gamma_{1}^{\prime}+\gamma_{2} \gamma_{2}^{\prime}, \quad F_{b} \propto \gamma_{1} \gamma_{2}^{\prime}+\gamma_{2} \gamma_{1}^{\prime}
$$

With account of the subtraction $\mathscr{K}_{G G}$ is presented in the form

$$
\frac{2 g^{4} N_{c}^{2}}{(2 \pi)^{D-1}} \hat{\mathscr{S}} \int_{0}^{1} d x \int \frac{d^{2+2 \varepsilon} k_{1}}{(2 \pi)^{D-1}}\left(\frac{a_{R} F_{a}\left(k_{1}, k_{2}\right)+b_{R} F_{b}\left(k_{1}, k_{2}\right)}{x(1-x)}\right)_{+},
$$

where $D=4+2 \varepsilon$ is the space-time dimension taken different from 4 to regularize infrared divergencies, the operator $\hat{\mathscr{S}}$ symmetrizes with respect to exchange of the Reggeon momenta, $x$ is a fraction of longitudinal momenta of a produced gluon,

$$
\left(\frac{f(x)}{x(1-x)}\right)_{+} \equiv \frac{1}{x}[f(x)-f(0)]+\frac{1}{(1-x)}[f(x)-f(1)] .
$$

The group coefficients are expressed through the coefficients $c_{R}$ appearing in the leading order: $a_{R}=c_{R}^{2}$ and $b_{R}=c_{R}\left(c_{R}-\frac{1}{2}\right)$. For the colour group $S U\left(N_{c}\right)$ with $N_{c}=3$ the possible representations $\mathscr{R}$ are

$$
\underline{1}, \underline{8_{a}}, \underline{8_{s}}, \underline{10}, \underline{\overline{10}}, \underline{27}
$$

Corresponding coefficients are

$$
c_{1}=1, c_{8_{a}}=c_{8_{s}}=\frac{1}{2}, c_{10}=c_{\overline{10}}=0, c_{27}=-\frac{1}{4 N_{c}} .
$$

In particular,

$$
a_{0}=1, a_{8_{a}}=a_{8_{s}}=\frac{1}{4}, b_{1}=1 / 2, b_{8_{a}}=b_{8_{s}}=0 .
$$

The last equality is especially important for the antisymmetric case, since the vanishing of $b_{8_{a}}$ is crucial for the gluon Reggeization. It means that only planar diagrams contribute to the colour octet kernel $\mathscr{K}_{G G}^{(8)}$. It simplifies extremely calculation of this kernel [6]. 
Instead of calculation of the second term in (3.3) we have found more convenient to calculate the "symmetric" contribution

$$
\mathscr{K}_{G G}^{(s)}\left(\vec{q}_{1}, \vec{q}_{2} ; \vec{q}\right)=\frac{2 g^{4} N_{c}^{2}}{(2 \pi)^{D-1}} \hat{\mathscr{S}} \int_{0}^{1} d x \int \frac{d^{2+2 \varepsilon} k_{1}}{(2 \pi)^{D-1}}\left(\frac{F_{s}\left(k_{1}, k_{2}\right)}{x(1-x)}\right)_{+},
$$

where

$$
F_{s}=F_{a}+F_{b} \propto\left(\gamma_{1}+\gamma_{2}\right)\left(\gamma_{1}^{\prime}+\gamma_{2}^{\prime}\right)
$$

A marvellous feature of $\mathscr{K}_{G G}^{(s)}$ is the absence of infrared singularities. The disappearance of the singularities is rather tricky: it takes place due to independence of infrared singular terms in $F_{s}$ from $x$. Because of this reason the singularities vanish after the substraction.

\subsection{Explicit representation of the kernel}

Relations between the colour coefficients $a_{R}$ and $b_{R}$ permits to write the two-gluon contribution to the kernel for any representation $\mathscr{R}$ is the form

$$
\mathscr{K}_{G G}^{(R)}=2 c_{R} \mathscr{K}_{G G}^{(8)}+b_{R} \mathscr{K}_{G G}^{(s)} .
$$

Moreover, in pure gluodynamics an analogous relations is valid for total "real" parts of the kernel:

$$
\mathscr{K}^{(R)}{ }_{r}=2 c_{R} \mathscr{K}^{(8)}{ }_{r}+b_{R} \mathscr{K}_{G G}^{(s)} .
$$

Since $\mathscr{K}_{G G}^{(s)}$ is infrared safe, this relation greatly simplifies analysis of infrared singularities, especially because the "real" part $\mathscr{K}^{(8)}{ }_{r}$ for the gluon channel is rather simple

$$
\begin{gathered}
\mathscr{K}_{r}^{(8)}\left(\vec{q}_{1}, \vec{q}_{2} ; \vec{q}\right)=\frac{g^{2} N_{c}}{2(2 \pi)^{D-1}}\left\{\left(\frac{\vec{q}_{1}^{2} \vec{q}_{2}^{\prime 2}+\vec{q}_{1}^{\prime 2} \vec{q}_{2}^{2}}{\vec{k}^{2}}-\vec{q}^{2}\right)\right. \\
\times\left(\frac{1}{2}+\frac{g^{2} N_{c} \Gamma(1-\varepsilon)\left(\vec{k}^{2}\right)^{\varepsilon}}{(4 \pi)^{2+\varepsilon}}\left(-\frac{11}{6 \varepsilon}+\frac{67}{18}-\zeta(2)+\varepsilon\left(-\frac{202}{27}+7 \zeta(3)+\frac{11}{6} \zeta(2)\right)\right)\right) \\
+\frac{g^{2} N_{c} \Gamma(1-\varepsilon)}{(4 \pi)^{2+\varepsilon}}\left[\vec { q } ^ { 2 } \left(\frac{11}{6} \ln \left(\frac{\vec{q}_{1}^{2} \vec{q}_{2}^{2}}{\vec{q}^{2} \vec{k}^{2}}\right)+\frac{1}{4} \ln \left(\frac{\vec{q}_{1}^{2}}{\vec{q}^{2}}\right) \ln \left(\frac{\vec{q}_{1}^{\prime 2}}{\vec{q}^{2}}\right)+\frac{1}{4} \ln \left(\frac{\vec{q}_{2}^{2}}{\vec{q}^{2}}\right) \ln \left(\frac{\vec{q}_{2}^{\prime 2}}{\vec{q}^{2}}\right)\right.\right. \\
\left.+\frac{1}{4} \ln ^{2}\left(\frac{\vec{q}_{1}^{2}}{\vec{q}_{2}^{2}}\right)\right)-\frac{\vec{q}_{1}^{2} \vec{q}_{2}^{\prime 2}+\vec{q}_{2}^{2} \vec{q}_{1}^{\prime 2}}{2 \vec{k}^{2}} \ln { }^{2}\left(\frac{\vec{q}_{1}^{2}}{\vec{q}_{2}^{2}}\right)+\frac{\vec{q}_{1}^{2} \vec{q}_{2}^{\prime 2}-\vec{q}_{2}^{2} \vec{q}_{1}^{\prime 2}}{\vec{k}^{2}} \ln \left(\frac{\vec{q}_{1}^{2}}{\vec{q}_{2}^{2}}\right)\left(\frac{11}{6}-\frac{1}{4} \ln \left(\frac{\vec{q}_{1}^{2} \vec{q}_{2}^{2}}{\vec{k}^{4}}\right)\right) \\
+\frac{1}{2}\left[\vec{q}^{2}\left(\vec{k}^{2}-\vec{q}_{1}^{2}-\vec{q}_{2}^{2}\right)+2 \vec{q}_{1}^{2} \vec{q}_{2}^{2}-\vec{q}_{1}^{2} \vec{q}_{2}^{\prime 2}-\vec{q}_{2}^{2} \vec{q}_{1}^{\prime 2}+\frac{\vec{q}_{1}^{2} \vec{q}_{2}^{\prime 2}-\vec{q}_{2}^{2} \vec{q}_{1}^{\prime 2}}{\vec{k}^{2}}\left(\vec{q}_{1}^{2}-\vec{q}_{2}^{2}\right)\right] \\
\left.\left.\times I\left(\vec{q}_{1}^{2}, \vec{q}_{2}^{2}, \vec{k}^{2}\right)\right]\right\}+\frac{g^{2} N_{c}}{2(2 \pi)^{D-1}}\left\{\vec{q}_{i} \longleftrightarrow \vec{q}_{i}^{\prime}\right\},
\end{gathered}
$$

where

$$
I(a, b, c)=\int_{0}^{1} \frac{d x}{a(1-x)+b x-c x(1-x)} \ln \left(\frac{a(1-x)+b x}{c x(1-x)}\right) .
$$

Due to infrared safety of $\mathscr{K}_{G G}^{(s)}$ the singularities are the same for all colour states in the $t$-channels, apart from colour factors. Actually the singularities are the same as for the forward case, since they 
are proportional to the LO kernel. For the Pomeron channel the total kernel $\hat{\mathscr{K}}=\hat{\omega}_{1}+\hat{\omega}_{2}+\hat{\mathscr{K}}_{r}$ must be infrared safe. In this case the singularities of $\mathscr{K}_{r}$ are cancelled by the singularities of the gluon trajectory. In [3] the infrared safety is explicitly demonstrated and forms free from the singularities are found.

The "symmetric" contribution is rather complicated. The complexity is related to the nonplanar diagrams. It is known since the calculation of the non-forward kernel for the QED Pomeron [9] where only box and cross-box diagrams are relevant. The kernel was found only in the form of two-dimensional integral. In QCD the situation is greatly worse because of existence of crosspentagon and cross-hexagon diagrams in addition to QED-type cross-box diagrams. It requires the use of additional Feynman parameters. At arbitrary $D$ no integration over these parameters at all can be done in elementary functions. It occurs, however, that in the limit $\varepsilon \rightarrow 0$ the integration over additional Feynman parameters can be performed, so that the result can be written as twodimensional integral, as well as in QED.

The result can be written as

$$
\begin{gathered}
\mathscr{K}_{G G}^{(s)}\left(\vec{q}_{1}, \vec{q}_{2} ; \vec{q}=\frac{\alpha_{s}^{2} N_{c}^{2}}{4 \pi^{3}}\left\{\left(\left[\left(\vec{q}^{2}-2 \vec{q}_{1}^{2}\right)\left(\frac{25}{9}-\frac{\pi^{2}}{12}\right)-\frac{11}{12}\left(2 \vec{q}_{1}^{2} \ln \left(\frac{\vec{q}_{1}^{2}}{\vec{k}^{2}}\right)-\vec{q}^{2} \ln \left(\frac{\vec{q}^{2}}{\vec{k}^{2}}\right)\right)\right.\right.\right.\right. \\
+\frac{\vec{q}^{2}}{4} \ln \left(\frac{\vec{q}_{1}^{2}}{\vec{q}^{2}}\right) \ln \left(\frac{\vec{q}_{1}^{\prime 2}}{\vec{q}^{2}}\right)-\frac{\vec{q}_{1}^{\prime 2}}{2}\left(\frac{\left(\vec{k}^{2}-\vec{q}_{1}^{2}-\vec{q}_{2}^{2}\right)^{2}-4 \vec{q}_{1}^{2} \vec{q}_{2}^{2}}{2 \vec{k}^{2}} I\left(\vec{k}^{2}, \vec{q}_{2}^{2}, \vec{q}_{1}^{2}\right)\right. \\
\left.\left.\left.\left.+\frac{\vec{k}^{2}+\vec{q}_{2}^{2}-\vec{q}_{1}^{2}}{2 \vec{k}^{2}} \ln \left(\frac{\vec{k}^{2}}{\vec{q}_{2}^{2}}\right) \ln \left(\frac{\vec{q}_{1}^{2}}{\vec{q}_{2}^{2}}\right)\right)-J\left(\vec{q}_{1}, \vec{q}_{2} ; \vec{q}\right)\right]+\left[\vec{q}_{i} \leftrightarrow-\vec{q}_{i}^{\prime}\right]\right)+\left(\vec{q}_{1} \leftrightarrow-\vec{q}_{2}^{\prime}\right)\right\},
\end{gathered}
$$

with the two-dimensional integral $J\left(\vec{q}_{1}, \vec{q}_{2} ; \vec{q}\right)$ :

$$
\begin{gathered}
J\left(\vec{q}_{1}, \vec{q}_{2} ; \vec{q}\right)=\int_{0}^{1} d x \int_{0}^{1} d z\left\{\vec{q}_{1} \vec{q}_{1}^{\prime}\left(\left(2-x_{1} x_{2}\right) \ln \left(\frac{Q^{2}}{\vec{k}^{2}}\right)-\frac{2}{x_{1}} \ln \left(\frac{Q^{2}}{Q_{0}^{2}}\right)\right)\right. \\
-\frac{1}{2 Q^{2}} x_{1} x_{2}\left(\vec{q}_{1}^{2}-2 \vec{q}_{1} \vec{p}_{1}\right)\left(\vec{q}_{1}^{\prime 2}-2 \vec{q}_{1}^{\prime} \vec{p}_{2}\right)+\frac{2}{x_{1}}\left[\left(x_{2} \vec{q}_{1} \vec{q}_{1}^{\prime}\left(\vec{p}_{1}\left(\vec{q}_{1}^{\prime}-\vec{p}_{2}\right)\right)-\vec{q}_{1}^{\prime 2} \vec{q}_{1} \vec{p}_{2}\right) \frac{1}{Q^{2}}\right. \\
\left.+\left(z(1-z) \vec{q}_{2}^{\prime 2} \vec{q}_{1} \vec{q}_{1}^{\prime}+\vec{q}_{1}^{\prime 2}\left(z \vec{q}_{1} \vec{k}+(1-z) \vec{q}_{1} \vec{q}_{1}^{\prime}\right)\right) \frac{1}{Q_{0}^{2}}\right]-\frac{1}{Q^{2}}\left(\vec{q}_{1}^{\prime 2} \vec{q}_{1}\left(\vec{p}_{1}-2 \vec{q}_{1}^{\prime}\right)\right. \\
\left.+4 x_{1} \vec{q}_{1}^{2}\left(\vec{q}_{1}^{\prime} \vec{p}_{2}\right)+\vec{q}_{1}^{\prime} \vec{q}_{1}\left(\vec{q}_{1}^{\prime} \vec{q}_{1}-\vec{q}_{1}^{\prime} \vec{p}_{1}-\vec{q}_{1} \vec{p}_{2}\right)+2\left(\vec{q}_{1}^{\prime} \vec{p}_{1}\right)\left(\vec{q}_{1} \vec{p}_{2}\right)-2\left(\vec{q}_{1}^{\prime} \vec{p}_{2}\right)\left(\vec{q}_{1} \vec{p}_{1}\right)\right) \\
+\vec{q}_{1}^{\prime 2}\left[\frac{-1}{\mu_{2}^{2} Q^{2}}\left(2 \frac{x_{2}}{x_{1}}\left(\vec{q}_{1} \vec{p}_{2}\right) \vec{q}_{1}^{\prime} \vec{k}+x_{2}\left(\vec{q}_{1}^{\prime} \vec{p}_{2}\right)\left(\vec{q}_{2}^{2}-\vec{k}^{2}\right)+2\left(\vec{q}_{2} \vec{p}_{2}\right) \vec{q}_{1} \vec{q}\right)\right. \\
+\frac{2}{\mu_{0}^{2} Q_{0}^{2}} \frac{1}{x_{1}}\left(\vec{q}_{1} \vec{p}_{0}\right) \vec{q}_{1}^{\prime} \vec{k}-\frac{\vec{q}_{1}\left(\vec{q}_{1}^{\prime}+\vec{k}_{1}\right)}{x_{1}}\left(\frac{x_{2}}{\vec{p}_{2}^{2}} \ln \left(\frac{Q^{2}}{\mu_{2}^{2}}\right)-\frac{1}{\vec{p}_{0}^{2}} \ln \left(\frac{Q_{0}^{2}}{\mu_{0}^{2}}\right)\right) \\
+\frac{1}{\vec{p}_{2}^{2}}\left(\frac{1}{\vec{p}_{2}^{2}} \ln \left(\frac{Q^{2}}{\mu_{2}^{2}}\right)+\frac{1}{Q^{2}}\right)\left(2 \frac{x_{2}}{x_{1}}\left(\vec{q}_{1} \vec{p}_{2}\right)\left(\vec{q}_{1}^{\prime}+\vec{k}\right) \vec{p}_{2}-2\left(\left(x_{2} \vec{q}_{1}^{\prime}+\vec{q}_{2}\right) \vec{p}_{2}\right) \vec{q}_{1} \vec{p}_{2}\right) \\
-\frac{1}{\vec{p}_{0}^{2}}\left(\frac{1}{\vec{p}_{0}^{2}} \ln \left(\frac{Q_{0}^{2}}{\mu_{0}^{2}}\right)+\frac{1}{Q_{0}^{2}}\right)\left(2 \frac{1}{x_{1}}\left(\vec{q}_{1} \vec{p}_{0}\right)\left(\vec{q}_{1}^{\prime}+\vec{k}\right) \vec{p}_{0}\right)+\frac{\left(x_{2} \vec{q}_{1}^{\prime}+\vec{q}_{2}\right) \vec{q}_{1}}{\vec{p}_{2}^{2}} \ln \left(\frac{Q^{2}}{\mu_{2}^{2}}\right)
\end{gathered}
$$




$$
\begin{gathered}
+\frac{\vec{q}_{1}^{2}}{d}\left(\left(\vec{q}_{2} \vec{k}\right)\left(\vec{q}_{2}^{\prime} \vec{k}\right)\left(\frac{Q^{2}}{d} \mathscr{L}-\frac{1}{\vec{k}^{2}}\right)+\left(\vec{q}_{2} \vec{p}_{2}\right)\left(\vec{q}_{2}^{\prime} \vec{k}\right)\left(\frac{1}{\mu_{2}^{2}}-\frac{\mu_{1}^{2}}{d} \mathscr{L}\right)+\left(\vec{q}_{2} \vec{k}\right)\left(\vec{q}_{2}^{\prime} \vec{p}_{1}\right)\left(\frac{1}{\mu_{1}^{2}}-\frac{\mu_{2}^{2}}{d} \mathscr{L}\right)\right. \\
\left.\left.\left.+\left(\vec{q}_{2} \vec{p}_{2}\right)\left(\vec{q}_{2}^{\prime} \vec{p}_{1}\right)\left(\frac{\vec{k}^{2}}{d} \mathscr{L}-\frac{1}{Q^{2}}\right)+\frac{\left(\vec{q}_{2} \vec{q}_{2}^{\prime}\right)}{2} \mathscr{L}\right)\right]\right\}
\end{gathered}
$$

Here

$$
\begin{gathered}
\vec{p}_{1}=z x \vec{q}_{1}+(1-z)\left(x \vec{k}-(1-x) \vec{q}_{2}^{\prime}\right), \quad \vec{p}_{2}=z\left((1-x) \vec{k}-x \vec{q}_{2}\right)+(1-z)(1-x) \vec{q}_{1}^{\prime} ; \\
Q^{2}=x(1-x)\left(\vec{q}_{1}^{2} z+\vec{q}_{1}^{\prime 2}(1-z)\right)+z(1-z)\left(\vec{q}_{2}^{2} x+\vec{q}_{2}^{\prime 2}(1-x)-\vec{q}^{2} x(1-x)\right), \\
\mu_{i}^{2}=Q^{2}+\vec{p}_{i}^{2}, \quad d=\mu_{1}^{2} \mu_{2}^{2}-\vec{k}^{2} Q^{2}, \quad \mathscr{L}=\ln \left(\frac{\mu_{1}^{2} \mu_{2}^{2}}{\vec{k}^{2} Q^{2}}\right) \\
\vec{p}_{0}=z \vec{k}+(1-z) \vec{q}_{1}^{\prime} ; \quad Q_{0}^{2}=z(1-z) \vec{q}_{2}^{\prime 2}, \quad \mu_{0}^{2}=z \vec{k}^{2}+(1-z) \vec{q}_{1}^{\prime 2} .
\end{gathered}
$$

\subsection{Analysis of the two-dimensional integral}

Presence of the two-dimensional integral $J\left(\vec{q}_{1}, \vec{q}_{2} ; \vec{q}\right)$ in the kernel makes difficult its use both for analytical investigation and for numerical calculations.

Unfortunately, the integrand INT $(x, z)$ of $J\left(\vec{q}_{1}, \vec{q}_{2} ; \vec{q}\right)$ is too complicated. Moreover, it's behaviour near the board of the integration region is not smooth. Analytical analysis of the integrand near the board can facilitate the numerical calculations. The most important are the corner regions [10]:

$x \ll 1, z \ll 1:$

$$
\begin{gathered}
\operatorname{INT}(x, z) \simeq \frac{2}{x}\left(\frac{\left(\vec{q}_{1} \vec{q}_{1}^{\prime}\right) \vec{q}_{1}^{\prime} \vec{q}_{2}^{\prime}}{\vec{q}_{1}^{\prime 2}}-\frac{\left(\vec{q}_{1} \vec{q}_{2}^{\prime}\right)}{2}\right) \ln \left(\frac{x \vec{q}_{1}^{\prime 2}+z \vec{q}_{2}^{\prime 2}}{z \vec{q}_{2}^{\prime 2}}\right)+\frac{1}{x \vec{q}_{1}^{\prime 2}+z \vec{q}_{2}^{\prime 2}} \\
\times\left[\vec{q}_{1}^{\prime 2}\left(\vec{q}_{2}^{2}-\vec{k}^{2}+\vec{q}_{1} \vec{q}_{2}^{\prime}\right)-\vec{q}_{1}^{2}\left(\vec{q}_{1}^{\prime} \vec{q}_{2}\right)+\left(\vec{q}_{1} \vec{q}_{1}^{\prime}\right)\left(2 \vec{q}_{1} \vec{q}_{1}^{\prime}+\vec{q}_{1}^{\prime} \vec{q}_{2}^{\prime}\right)+2\left(\vec{q}_{1}^{\prime} \vec{q}_{2}\right)\left(\vec{q}_{1} \vec{q}\right)\right] .
\end{gathered}
$$

$x \ll 1,1-z \ll 1$ :

$$
\begin{gathered}
\operatorname{INT}(x, z) \simeq \frac{2}{x}\left[\vec{q}_{1} \vec{q}_{1}^{\prime}-\vec{q}_{1}^{\prime 2}\left(\frac{\left(\vec{q}_{1} \vec{k}\right)\left(\vec{q}_{1}^{\prime} \vec{k}\right)}{\vec{k}^{4}}-\frac{\left(\vec{q}_{1} \vec{q}_{2}^{\prime}\right)}{2 \vec{k}^{2}}\right)\right] \ln \left(\frac{x \vec{q}_{1}^{2}+(1-z) \vec{q}_{2}^{\prime 2}}{(1-z) \vec{q}_{2}^{\prime 2}}\right) \\
+\frac{1}{x \vec{q}_{1}^{2}+(1-z) \vec{q}_{2}^{\prime 2}}\left[-2 \vec{q}_{1}^{\prime 2}\left(\vec{q}_{1} \vec{q}\right)-\left(\vec{q}_{1} \vec{q}_{1}^{\prime}\right)\left(2 \vec{q}_{1}^{\prime 2}+2 \vec{q}_{1}^{2}+\vec{q}_{1} \vec{q}_{2}^{\prime}\right)\right. \\
+\frac{\vec{q}_{1}^{\prime 2}}{\vec{k}^{2}}\left(2\left(\vec{q}_{1} \vec{k}\right)^{2}+2\left(\vec{q}_{1} \vec{k}\right)\left(\vec{q}_{1}^{2}+\vec{q}_{1} \vec{q}_{2}\right)+\vec{q}_{1}^{2}\left(\vec{q}_{1}^{\prime} \vec{k}\right)+\frac{\vec{q}_{1}^{2}\left(\vec{q}_{2} \vec{k}\right)\left(x \vec{q}_{1} \vec{q}_{2}^{\prime}-(1-z) \vec{q}_{2}^{\prime 2}\right)}{x \vec{q}_{1}^{2}+(1-z) \vec{q}_{2}^{\prime 2}}\right) \\
\left.+\frac{\vec{q}_{1}^{2} \vec{q}_{1}^{\prime 2}}{\vec{k}^{2}}\left(\frac{\left(\vec{q}_{2} \vec{k}\right)\left(\vec{q}_{2}^{\prime} \vec{k}\right)}{\vec{k}^{\prime 2}}-\frac{\left(\vec{q}_{2} \vec{q}_{2}^{\prime}\right)}{2}\right)\right] .
\end{gathered}
$$

$1-x \ll 1, z \ll 1:$

$$
\operatorname{INT}(x, z) \simeq \frac{1}{(1-x) \vec{q}_{1}^{\prime 2}+z \vec{q}_{2}^{2}}\left[\left(\vec{q}_{1} \vec{q}_{1}^{\prime}\right)\left(\vec{q}_{2} \vec{q}_{1}^{\prime}\right)+\vec{q}_{1}^{\prime 2}\left(\vec{q}_{1}^{2}-2 \vec{q}_{1} \vec{q}_{1}^{\prime}\right)\right.
$$




$$
\left.+\vec{q}_{1}^{2}\left(\frac{\left(\vec{q}_{2} \vec{k}\right)\left(\vec{q}_{2}^{\prime} \vec{k}\right)}{\vec{k}^{4}}-\frac{\left(\vec{q}_{2} \vec{q}_{2}^{\prime}\right)}{2 \vec{k}^{2}}\right)\right]+\frac{(1-x) \vec{q}_{1}^{\prime} \vec{q}_{2}-z \vec{q}_{2}^{2}}{\left((1-x) \vec{q}_{1}^{\prime 2}+z \vec{q}_{2}^{2}\right)^{2}} \vec{q}_{1}^{\prime 2}\left(2 \vec{q}_{1} \vec{q}+\vec{q}_{1}^{2} \frac{\vec{q}_{2}^{\prime} \vec{k}}{\vec{k}^{2}}\right) .
$$

$1-x \ll 1,1-z \ll 1$ :

$$
\operatorname{INT}(x, z) \simeq \frac{\left[-\vec{q}_{1}^{\prime 2}\left(\vec{q}_{1}\left(\vec{q}_{1}^{\prime}+\vec{q}_{2}\right)\right)-2 \vec{q}_{1}^{2}\left(q_{1}^{\prime} \vec{q}_{2}\right)-\left(\vec{q}_{1} \vec{q}_{1}^{\prime}\right)\left(\vec{q}_{1} \vec{q}_{2}\right)\right]}{(1-x) \vec{q}_{1}^{2}+(1-z) \vec{q}_{2}^{2}} .
$$

Contributions of the corner regions are calculated analytically. Unfortunately, to do it for the regions where one of the variables is closed to the board whereas another is arbitrary, is practically impossible, although the integrand in these regions is also found [10].

\subsection{Alternative representation}

Because of complexity of the integral (3.15) another representations of $\mathscr{K}_{G G}^{(s)}$ are desirable. The representation in the form of the integral in the transverse momentum space [10] can be useful both for analytical investigation and numerical integration:

$$
\mathscr{K}_{G G}^{(s)}\left(\vec{q}_{1}, \vec{q}_{2} ; \vec{q}\right)=\frac{\alpha_{s}^{2} N_{c}^{2}}{4 \pi^{3}}\left(\left[J^{s}\left(\vec{q}_{1}, \vec{q}_{2} ; \vec{q}\right)+J^{s}\left(-\vec{q}_{2},-\vec{q}_{1} ;-\vec{q}\right)\right]+\left[\vec{q}_{n} \leftrightarrow \vec{q}-\vec{q}_{n}\right]\right),
$$

where $\vec{q}_{n}$ and $\vec{q}-\vec{q}_{n} \equiv-\vec{q}_{n}^{\prime}(n=1,2)$ are the $t$-channel Reggeized gluon momenta,

$$
\begin{gathered}
J^{s}\left(\vec{q}_{1}, \vec{q}_{2} ; \vec{q}\right)=\frac{\vec{k}^{2}}{2}+\frac{\vec{q}^{2}}{2}\left(\frac{13}{18}-\zeta(2)\right)-\frac{\left(\vec{q}_{1}^{2}-\vec{q}_{2}^{2}\right)\left(\vec{q}_{1}^{\prime 2}-\vec{q}_{2}^{\prime 2}\right)}{2 \vec{k}^{2}}-\vec{q}^{2}\left(\frac{11}{12} \ln \left(\frac{\vec{q}^{2}}{\vec{k}^{2}}\right)+\frac{5}{6} \ln 2\right) \\
+2\left(\vec{q}_{1} \vec{q}_{2}-\vec{q}_{1}^{2} \frac{\vec{k}_{1}^{\prime}}{\vec{k}^{2}}\right) \ln \left(\frac{\vec{q}_{1}^{2}}{\vec{k}^{2}}\right)-\frac{\vec{k}^{2}}{2} \ln \left(\frac{\vec{q}_{1}^{2}}{\vec{k}^{2}}\right) \ln \left(\frac{\vec{q}_{2}^{2}}{\vec{k}^{2}}\right)+\frac{\vec{q}^{2}}{4} \ln \left(\frac{\vec{q}_{1}^{2}}{\vec{q}^{2}}\right) \ln \left(\frac{\vec{q}_{1}^{\prime 2}}{\vec{q}^{2}}\right) \\
\left.+\left(\vec{q}_{1} \vec{q}+\frac{\vec{q}_{1}^{2}\left(\vec{k} \vec{q}_{2}^{\prime}\right)-\vec{q}_{1}^{\prime 2}\left(\vec{k} \vec{q}_{2}\right)}{\vec{k}^{2}}\right)\left(\frac{1}{2} \ln \left(\frac{\vec{q}_{2}^{2}}{\vec{k}^{2}}\right) \ln \left(\frac{\vec{q}_{1}^{2}}{\vec{q}_{2}^{2}}\right)+\left(\vec{q}_{2} \vec{k}\right) I\left(\vec{q}_{1}^{2}, \vec{q}_{2}^{2}, \vec{k}^{2}\right)\right)\right) \\
+\frac{5}{2}\left(\vec{q}_{1} \vec{q}_{2}\right)-\vec{q}_{2}^{2}\left(\vec{q}_{1}^{\prime} \vec{q}\right) I\left(\vec{q}_{1}^{2}, \vec{q}_{2}^{2}, \vec{k}^{2}\right)+\int \frac{d^{2} k_{1}}{\pi}\left[\left(-\frac{\vec{k}_{1}^{2} \vec{k}_{2}^{2}}{2}+\left(\vec{k}_{1} \vec{k}_{2}\right)^{2}+\left(Q_{1}^{i} \Omega_{1}^{i j} Q_{2}^{\prime j}\right)\left(Q_{1}^{i} \Omega_{2}^{i j} Q_{2}^{\prime j}\right)\right.\right. \\
\left.\left.-\vec{Q}_{2}^{\prime 2}\left(Q_{1}^{i} \Omega_{1}^{i j} \Omega_{2}^{j l} Q_{1}^{l}\right)\right) \times \frac{1}{\vec{Q}_{1}^{2} \vec{Q}_{2}^{\prime 2}-\vec{k}_{1}^{2} \vec{k}_{2}^{2}} \ln \left(\frac{\vec{Q}_{1}^{2} \vec{Q}_{2}^{\prime 2}}{\vec{k}_{1}^{2} \vec{k}_{2}^{2}}\right)-\frac{1}{2}+\frac{5}{6} \frac{\vec{q}^{2}}{\vec{k}_{1}^{2}+\vec{k}_{2}^{2}}\right] .
\end{gathered}
$$

Here $\vec{k}=\vec{q}_{1}-\vec{q}_{2}=\vec{q}_{1}^{\prime}-\vec{q}_{2}^{\prime}, \vec{k}_{2}=\vec{k}-\vec{k}_{1}, \vec{Q}_{n}=\vec{q}_{1}-\vec{k}_{n}, \vec{Q}_{n}^{\prime}=\vec{q}_{1}^{\prime}-\vec{k}_{n}, \Omega_{n}^{i j}=\delta^{i j}-2 k_{n}^{i} k_{n}^{i} / \vec{k}_{n}^{2}$.

\section{BFKL in coordinate representation}

In the following only the colour singlet channel is considered. There are at least two reasons for consideration of the singlet BFKL kernel in the coordinate representation in the transverse space. First, just in this representation the BFKL equation in the leading approximation exhibits the famous property of conformal invariance [11], which is extremely important for finding solutions of the equation. Second, it is the representation in which the color dipole approach to high energy scattering [12], very popular now, is formulated. An advantage of this approach is a clear physical picture of the high energy processes. Moreover, this approach is naturally applied not only at low 
parton densities, but in the saturation regime [13], where equations of evolution of parton densities with energy become nonlinear. Examination of the BFKL kernel in the coordinate representation is needed for understanding its conformal properties and relation between the BFKL and the colour dipole approaches. A clear understanding of this relation is very important. It could help in further development of the theoretical description of small-x processes. It was affirmed [12] with the advent of the dipole approach that in the linear regime it gives the same results as the BFKL approach for the colour singlet channel. The relation between the BFKL and colour dipole in the leading order was analyzed recently in [14]. This analysis is extended in [15] at NLO. It is based on direct transformation of the BFKL kernel from the momentum representation to the coordinate representation. Since the NLO calculations are performed using the dimensional regularization, the space-time dimension $D=4+2 \varepsilon$ is used in the LO as well, with the result

$$
\begin{gathered}
\left\langle\vec{r}_{1}, \vec{r}_{2}|\hat{\mathscr{K}}| \vec{r}_{1}^{\prime}, \vec{r}_{2}^{\prime}\right\rangle=\left\langle\vec{r}_{1}, \vec{r}_{2}\left|\hat{\mathscr{K}}_{d}\right| \vec{r}_{1}^{\prime}, \vec{r}_{2}^{\prime}\right\rangle-\frac{g^{2} N_{c} \Gamma^{2}(1+\varepsilon)}{8 \pi^{3+2 \varepsilon}} \\
\times\left[\frac{\delta\left(\vec{r}_{1}-\vec{r}_{1}^{\prime}\right)}{\left(\vec{r}_{1}-\vec{r}_{2}^{\prime}\right)^{2(1+2 \varepsilon)}}+\frac{\delta\left(\vec{r}_{2}-\vec{r}_{2}^{\prime}\right)}{\left(\vec{r}_{2}-\vec{r}_{1}^{\prime}\right)^{2(1+2 \varepsilon)}}-2 \frac{\delta\left(\vec{r}_{1}^{\prime}-\vec{r}_{2}^{\prime}\right)\left(\vec{r}_{1}-\vec{r}_{1}^{\prime}\right)\left(\vec{r}_{2}-\vec{r}_{2}^{\prime}\right)}{\left(\vec{r}_{1}-\vec{r}_{1}^{\prime}\right)^{2(1+\varepsilon)}\left(\vec{r}_{2}-\vec{r}_{2}^{\prime}\right)^{2(1+\varepsilon)}}\right],
\end{gathered}
$$

where

$$
\begin{gathered}
\left\langle\vec{r}_{1}, \vec{r}_{2}\left|\hat{\mathscr{K}}_{d}\right| \vec{r}_{1}^{\prime}, \vec{r}_{2}^{\prime}\right\rangle=\frac{g^{2} N_{c} \Gamma^{2}(1+\varepsilon)}{8 \pi^{3+2 \varepsilon}} \int d^{2+2 \varepsilon} \rho\left(\frac{\left(\vec{r}_{1}-\vec{\rho}\right)}{\left(\vec{r}_{1}-\vec{\rho}\right)^{2(1+\varepsilon)}}-\frac{\left(\vec{r}_{2}-\vec{\rho}\right)}{\left(\vec{r}_{2}-\vec{\rho}\right)^{2(1+\varepsilon)}}\right)^{2} \\
\times\left(\delta\left(\vec{r}_{1}-\vec{r}_{1}^{\prime}\right) \delta\left(\vec{r}_{2}^{\prime}-\vec{\rho}\right)+\delta\left(\vec{r}_{2}-\vec{r}_{2}^{\prime}\right) \delta\left(\vec{r}_{1}^{\prime}-\vec{\rho}\right)-\delta\left(\vec{r}_{1}-\vec{r}_{1}^{\prime}\right) \delta\left(\vec{r}_{2}-\vec{r}_{2}^{\prime}\right)\right)
\end{gathered}
$$

is just the dipole kernel in $D$-2-dimensional space. It is seen from (4.1) that, strictly speaking, the BFKL and dipole kernels are not equivalent. However, if we consider scattering of colourless particles, there is a freedom in a choice of the kernel [11], [14] due to the "gauge invariance" of impact factors of such particles (vanishing of the impact factors at zero transverse momenta of Reggeons). The freedom permits to omit the terms in the square bracket.

So, the BFKL and dipole kernels are not related by the Fourier transform, i.e. they have only a "limited equivalence". Corresponding Green's functions are different, and only scattering amplitudes of colourless particles are the same.

In the momentum representation the kernels are related as

$$
\left\langle\vec{q}_{1} \vec{q}_{2}|\hat{\mathscr{K}}| \vec{q}_{1}^{\prime} \vec{q}_{2}^{\prime}\right\rangle=\left\langle\vec{q}_{1} \vec{q}_{2}\left|\hat{\mathscr{K}}_{d}\right| \vec{q}_{1}^{\prime} \vec{q}_{2}^{\prime}\right\rangle-\delta\left(\vec{q}-\vec{q}^{\prime}\right)\left[\delta\left(\vec{q}_{2}\right) \omega\left(\vec{q}_{2}^{\prime}\right)+\delta\left(\vec{q}_{1}\right) \omega\left(\vec{q}_{1}^{\prime}\right)+\frac{g^{2} N_{c}}{(2 \pi)^{3+2 \varepsilon}} \frac{2 \vec{q}_{1} \vec{q}_{2}}{\vec{q}_{1}^{2} \vec{q}_{2}^{2}}\right] .
$$

This equation can be obtained by the direct transformation of (4.2) in the momentum representation. Note, that it means

$$
\int d^{D-2} q_{1} d^{D-2} q_{2}\left\langle\vec{q}_{1} \vec{q}_{2}\left|\hat{\mathscr{K}}_{d}\right| \vec{q}_{1}^{\prime} \vec{q}_{2}^{\prime}\right\rangle=0
$$

i.e. that the dipole kernel does not satisfy to the "bootstrap condition"

$$
\int d^{D-2} q_{1} d^{D-2} q_{2}\left\langle\vec{q}_{1} \vec{q}_{2}\left|\left(\hat{\mathscr{K}}+\hat{\omega}_{1}+\hat{\omega}_{2}-2 \omega(t)\right)\right| \vec{q}_{1}^{\prime} \vec{q}_{2}^{\prime}\right\rangle=0,
$$

which is required for compatibility of the gluon Reggeizatiion (the basis of the BFKL approach) with the $s$ - channel unitarity. 
In the NLO with account of the freedom discussed above the renormalized BFKL kernel in the coordinate representation can be written as

$$
\begin{gathered}
\left\langle\vec{r}_{1} \vec{r}_{2}|\hat{\mathscr{K}}| \vec{r}_{1}^{\prime} \vec{r}_{2}^{\prime}\right\rangle=a_{r}\left[\delta\left(\vec{r}_{1}-\vec{r}_{1}^{\prime}\right) \delta\left(\vec{r}_{2}-\vec{r}_{2}^{\prime}\right) \int d^{D-2} \rho \bar{f}\left(\vec{r}_{1}, \vec{r}_{2} ; \rho\right)\right. \\
\left.+\delta\left(\vec{r}_{1}-\vec{r}_{1}^{\prime}\right) \bar{f}_{1}\left(\vec{r}_{1}, \vec{r}_{2} ; \vec{r}_{2}^{\prime}\right)+\delta\left(\vec{r}_{2}-\vec{r}_{2}^{\prime}\right) \bar{f}_{2}\left(\vec{r}_{1}, \vec{r}_{2} ; \vec{r}_{1}^{\prime}\right)+\frac{\Gamma(1+\varepsilon)}{2 \pi^{1+\varepsilon}} \bar{f}\left(\vec{r}_{1}, \vec{r}_{2} ; \vec{r}_{1}^{\prime}, \vec{r}_{2}^{\prime}\right)\right] .
\end{gathered}
$$

If we restrict ourselves to quark contribution at large $N_{c}$ in the limit $\varepsilon \rightarrow 0$, we have in the $\overline{M S}$ scheme

$$
\begin{gathered}
\bar{f}\left(\vec{r}_{1}, \vec{r}_{2} ; \vec{\rho}\right)=\frac{\left(\vec{r}_{1}-\vec{\rho}\right)\left(\vec{r}_{2}-\vec{\rho}\right)}{\left(\vec{r}_{2}-\vec{\rho}\right)^{2}\left(\vec{r}_{1}-\vec{\rho}\right)^{2}} \ln \left(\frac{\vec{r}_{\mu}^{4}}{\left(\vec{r}_{1}-\vec{\rho}\right)^{2}\left(\vec{r}_{2}-\vec{\rho}\right)^{2}}\right) \\
-\frac{1}{\left(\vec{r}_{1}-\vec{\rho}\right)^{2}} \ln \left(\frac{\vec{r}_{\mu}^{2}}{\left(\vec{r}_{1}-\vec{\rho}\right)^{2}}\right)-\frac{1}{\left(\vec{r}_{2}-\vec{\rho}\right)^{2}} \ln \left(\frac{\vec{r}_{\mu}^{2}}{\left(\vec{r}_{2}-\vec{\rho}\right)^{2}}\right), \\
\bar{f}_{1}\left(\vec{r}_{1}, \vec{r}_{2} ; \vec{\rho}\right)=-2 \frac{\left(\vec{r}_{1}-\vec{\rho}\right)\left(\vec{r}_{2}-\vec{\rho}\right)}{\left(\vec{r}_{1}-\vec{\rho}\right)^{2}\left(\vec{r}_{2}-\vec{\rho}\right)^{2}} \ln \left(\frac{\vec{r}_{\mu}^{2}}{\left(\vec{r}_{1}-\vec{\rho}\right)^{2}}\right)+\frac{1}{\left(\vec{r}_{2}-\vec{\rho}\right)^{2}} \ln \left(\frac{\vec{r}_{\mu}^{2}}{\left(\vec{r}_{2}-\vec{\rho}\right)^{2}}\right) \\
\frac{1}{\left(\vec{r}_{1}-\vec{\rho}\right)^{2}} \ln \left(\frac{\vec{r}_{\mu}^{2}}{\left(\vec{r}_{1}-\vec{\rho}\right)^{2}}\right)+\frac{1}{\left(\vec{r}_{1}-\vec{\rho}\right)^{2}} \ln \left(\frac{\left(\vec{r}_{2}-\vec{\rho}\right)^{2}}{\left(\vec{r}_{1}-\vec{r}_{2}\right)^{2}}\right)+\frac{\left(\vec{r}_{1}-\vec{\rho}\right)\left(\vec{r}_{2}-\vec{\rho}\right)}{\left(\vec{r}_{1}-\vec{\rho}\right)^{2}\left(\vec{r}_{2}-\vec{\rho}\right)^{2}} \ln \left(\frac{\left(\vec{r}_{1}-\vec{r}_{2}\right)^{2}}{\left(\vec{r}_{1}-\vec{\rho}\right)^{2}}\right), \\
\bar{f}\left(\vec{r}_{1}, \vec{r}_{2} ; \vec{r}_{1}^{\prime}, \vec{r}_{2}^{\prime}\right)=\frac{1}{\left(\vec{r}_{2}-\vec{r}_{2}^{\prime}\right)^{2}}\left[\frac{\left(\vec{r}_{1}-\vec{r}_{2}\right)^{2}}{\left(\vec{r}_{1}-\vec{r}_{2}^{\prime}\right)^{2}}\left(\frac{1}{\left(\vec{r}_{1}^{\prime}-\vec{r}_{2}^{\prime}\right)^{2}}-\frac{1}{\left(\vec{r}_{1}-\vec{r}_{1}^{\prime}\right)^{2}}\right)\right. \\
\left.+\frac{1}{\left(\vec{r}_{1}^{\prime}-\vec{r}_{2}^{\prime}\right)^{2}}\left(\frac{\left(\vec{r}_{2}-\vec{r}_{1}^{\prime}\right)^{2}}{\left(\vec{r}_{1}-\vec{r}_{1}^{\prime}\right)^{2}}-1\right)\right]+1 \leftrightarrow 2, \\
a_{r}=\frac{\alpha_{s}^{2}(\mu) N_{c} n_{f}}{12 \pi^{3}}, \ln \vec{r}_{\mu}^{2}=-\frac{5}{3}+2 \psi(1)-\ln \left(\frac{\mu^{2}}{4}\right) .
\end{gathered}
$$

It is seen that the conformal invariance is violated not only by the renormalization. We see also that the result (4.6)-(4.10) does not agree with the result obtained recently in [16] by direct calculation of quark contribution to the dipole kernel in the coordinate representation.

\section{Summary}

The BFKL kernel is known now for $t \neq 0$ and all possible $t$-channel colour states $\mathscr{R}$. It is expressed in terms of the gluon trajectory, the kernel in the octet channel and the "symmetric" contribution, which is infrared safe. It makes simple the infrared structure of the kernel for any $\mathscr{R}$ and evident the infrared safety of the singlet kernel. However "symmetric" contribution contains the two-dimensional integral with a complicated integrand. Analysis of the integrand in the nearboard region is performed. Alternative representation of the "symmetric" contribution is found. Work on search of suitable representations for the kernel and on investigation of its properties is continuing. The coordinate representation for the BFKL kernel is particularly interesting, because it 
gives the possibility to understand its conformal properties and relation between the BFKL and the colour dipole approaches. Unfortunately, transformation of the BFKL kernel from the momentum to the coordinate representation shows that violation of the conformal invariance in the NLO is not reduced to the renormalization. Then, the result of the transformation does not agree with the direct calculation of the dipole kernel in the NLO in the coordinate representation.

\section{References}

[1] V.S. Fadin, E.A. Kuraev and L.N. Lipatov, Phys. Lett. B 60 (1975) 50; E.A. Kuraev, L.N. Lipatov and V.S. Fadin, Zh. Eksp. Teor. Fiz. 71 (1976) 840 [Sov. Phys. JETP 44 (1976) 443 ]; 72 (1977) 377 [45 (1977) 199 ]; Ya.Ya. Balitskii and L.N. Lipatov, Sov. J. Nucl. Phys. 28 (1978) 822.

[2] V.S. Fadin, L.N. Lipatov, Phys. Lett. B 429 (1998) 127; M. Ciafaloni and G. Camici, Phys. Lett. B 430 (1998) 349.

[3] V.S. Fadin and R. Fiore, Phys. Lett. B 610 (2005) 61 [Erratum-ibid. B 621 (2005) 61]; Phys. Rev. D 72 (2005) 014018.

[4] V.S. Fadin and R. Fiore, Phys. Lett. B 440359 (1998).

[5] V.S. Fadin, Zh. Eksp. Teor. Fiz. Pis'ma 61342 (1995) ; V.S. Fadin, R. Fiore and A. Quartarolo, Phys. Rev. D 532729 (1996); M.I.Kotsky and V.S. Fadin, Yad. Fiz. 59(6) 1080 (1996); V.S. Fadin, R. Fiore and M.I. Kotsky, Phys. Lett. B 359181 (1995); V.S. Fadin, R. Fiore and M.I. Kotsky, Phys. Lett. B 387593 (1996); J. Blumlein, V. Ravindran and W. L. van Neerven, Phys. Rev. D 58091502 (1998); V. Del Duca and E. W. N. Glover, JHEP 0110035 (2001).

[6] V.S. Fadin and D.A. Gorbachev, Pis'ma v Zh. Eksp. Teor. Fiz. 71 (2000) 322 [JETP Letters 71 (2000) 222]; Phys. Atom. Nucl. 63 (2000) 2157 [Yad. Fiz. 63 (2000) 2253].

[7] V.S. Fadin, R. Fiore and A. Papa, Phys. Rev. D 60, 074025 (1999).

[8] L.N. Lipatov and V.S. Fadin, JETP Lett. 49 (1989) 352 [Yad. Fiz. 50 (1989 SJNCA,50,712.1989) $1141]$.

[9] H. Cheng, T.T. Wu, Phys. Rev. D 10 (1970) 2775; V.N. Gribov, L.N. Lipatov and G.V. Frolov, Sov. J. Nucl. Phys. 12 (1971) 543 [Yad. Fiz. 12 (1970) 994].

[10] V.S. Fadin and R. Fiore, to be published.

[11] L.N. Lipatov, Sov. Phys. JETP 63 (1986) 904 [Zh. Eksp. Teor. Fiz. 90 (1986) 1536].

[12] N.N. Nikolaev and B.G. Zakharov, Z. Phys. C 64 (1994) 631; N.N. Nikolaev, B.G. Zakharov and V.R. Zoller, JETP Lett. 59 (1994) 6; A.H. Mueller, Nucl. Phys. B 415 (1994) 373; A.H. Mueller and B. Patel, Nucl. Phys. B 425 (1994) 471.

[13] L.V. Gribov, E.M. Levin and M.G. Ryskin, Phys. Rep. 176 (1983) 1.

[14] J. Bartels, L.N. Lipatov and G.P. Vacca, Nucl. Phys. B 706 (2005) 391; J. Bartels, L.N. Lipatov, M.Salvadore and G.P. Vacca, Nucl. Phys. B 726 (2005) 53.

[15] V.S. Fadin, R. Fiore and A. Papa, to be published.

[16] I. Balitsky, arXiv:hep-ph/0609105. 